\title{
A Multimodal Approach to Translation Quality Assessment of Interlingual Subtitling: Theoretical Reflections
}

\author{
Zhen $\mathrm{Hu}^{1, *}$ \\ ${ }^{1}$ College of Foreign Languages and Cultures, Xiamen University, Xiamen, Fujian, China \\ *Corresponding author. Email: william2568886@gmail.com
}

\begin{abstract}
Featuring prominently in audiovisual products, translated subtitles have even become omnipresent in everyday life. The buoyancy of the demand has triggered in industry and academia a relentless pursuit of a well-founded translation quality assessment model for pre-recorded interlingual subtitling. Thus far, a significant number of translation quality assessment studies have addressed monomodal written texts. However, few efforts have been channeled into the translation quality assessment of subtitle translation, not to mention assumed a linguistic (multimodal) approach to develop a fully-fledged and commonly accepted framework in this field. As an initial theoretical attempt, this paper takes into consideration the multimodal characteristics of audiovisual texts and argues that it is possible to integrate the existing translation quality assessment models for monomodal texts and the analytical scheme of multimodal translation to evaluate the translation quality of interlingual subtitling. In this way, the current research moves a step further towards optimizing translation quality assessment models for interlingual subtitling and lays a theoretical foundation for the potential emergence of the theoretical model that can offer insights for subtitlers on furnishing audiences with better translations.
\end{abstract}

Keywords: Interlingual subtitling, Translation quality assessment, Systemic Functional Linguistics, Multimodality, Audiovisual translation

\section{INTRODUCTION}

With the growing significance of audiovisual translation both in the field of translation studies and applied linguistics, as well as the ever-increasing importance of subtitling in the audiovisual production world, a high premium should be placed on the translation quality of subtitles in audiovisual textualities, especially interlingual subtitling (subtitle translation between different languages) for movies and television shows, whose worldwide proliferation has mushroomed over the past decade. However, notwithstanding its significant role, few efforts have been made to delve into the translation quality assessment (TQA) of interlingual subtitling, and a fortiori take a linguistic (multimodal) approach toward developing a full-fledged framework in this realm (though audiovisual materials are predominantly multimodal).

As one of the most contentious and recurring topics in translation studies $[1,2]$, TQA, sometimes termed translation (quality) evaluation [3] or translation criticism [3], is a statement of translation quality that touches upon assessing the worth of translation retrospectively by examining the linkage between a source text and its translation on the premise of a comparative analytical evaluation $[2,4]$.

Although these three terms are used synonymously from time to time, it is pertinent to elucidate their distinctions. By McAlester's definition [3], translation evaluation means that some value is attached to translation, namely in the form of grading. Whatever be the result, it should be warranted by certain normative procedures, although it is the final value that is normally emphasized in the translation evaluation. By contrast, translation criticism refers to scrutinizing the appropriateness of a translation from social and cultural perspectives [5], which can involve (but not necessarily) a quantified examination (i.e., giving a mark). Though such an evaluation is typically not mandatory, translation criticism, ipso facto, implies a value judgment [3]. Hence, 
as claimed by some scholars, translation evaluation can be viewed as a constituent of translation criticism [5]. As stated, although translation evaluation and criticism each lay different emphasis on the end product (of assessment), they do share considerable similarities in the process. Ergo, it is not essential to differentiate between these two expressions for this study, which treats them as two sides of the same coin. The working definition of TQA is the composite of a value statement and the (optional) qualitative analysis of a translation. Notwithstanding the already discussed difference between the concept of evaluation and assessment, it deserves to be noted that in this study, the verbs assess, appraise, and evaluate, and similarly, their agent nouns [3], i.e., assessor and evaluator, are all used interchangeably without variations in meaning.

Although numerous models deploying a multitude of indicators with a blizzard of purposes and criteria have been proposed to assess translation quality [6], they are not without their drawbacks. Unfortunately, most of them have failed to achieve broad acceptance and have not enjoyed practical applicability in the translation industry [1].

Despite the equivocal nature of translation quality and the fact that subjectivity is deeply ingrained in most assessment models (and concomitant debatable reliability), TQA, as one of the most vital procedures in every translation task, is carried out at a large scale in the translation industry by proofreaders and translators, who sometimes reckon with readers' feedback (which can constitute a measure of TQA). Interlingual subtitling does not constitute an exception in any way. Subtitle translation is described as "vulnerable translation" [7], because having developed critical consciousness of subtitle quality, even some end-users (the audience) may appraise translation quality from pluralistic perspectives while reading subtitles contained in multimedia products (such as DVDs and Blu-rays), not to mention the subtitlers themselves. Both the professional and academic world has witnessed the increasing necessity of objective (to the extent possible) measures of the TQA of interlingual subtitling, which is engendered by the mounting demand for high-quality subtitle translation service in a world where subtitling is prevalent. Subtitle translation quality (as a product) is a sine qua non and the touchstone of audiovisual translation studies.

Up to now, an appreciable number of studies have been conducted to test the validity of existing (empirical) TQA schemes, which are established on the basis of any explicit linguistic theory. In contradistinction to the burgeoning empirical research, failing to gain enough ground within TQA studies, the construction of a schematic and theoretical TQA model for interlingual subtitling has been largely under-researched.

\section{LITERATURE REVIEW}

Thus far, some studies have been focused on TQA of subtitling, a part of which have involved rudimentary discussions on the determinants of subtitle translation quality from the perspective of subtitling as a professional practice [8] or have dealt with the quality of machine translation and post-editing work around subtitles [9]. However, such research has not significantly contributed to the TQA of human-translated (vs. machine-translated) subtitles.

Significantly, targeting interlingual human-translated subtitling squarely, the clearly structured FAR model [10] is a comparatively functional one that assesses the quality of subtitles from three perspectives: functional equivalence, acceptability, and readability. It gains insight from national subtitling norms (see [10] for more detail) and takes an experience-based approach [1]. It may be delineated as an inclusive model as it not only handles linguistic problems (functional equivalence and acceptability) but also takes account of technical issues (readability), such as spotting, line length, etc. Its universality is guaranteed because it neither imposes restrictions on the genre of the subjects nor does it specify the language pair(s) in comparison. Nonetheless, similar to its predecessors, the FAR model follows the trend in forming a system of marking scales (the point deduction system) in recent TQA research. Therefore, it deals with translation problems in a traditional manner, namely by counting grammatical and semantic mistakes and establishing a hierarchy of errors, e.g., minor, standard, and serious (or severe) ones, which can be traced to measures postulated by Williams [11]. The attractiveness of such a system lies in its simplicity [11]. The model is not demanding for evaluators to handle, which ensures its adaptability in the subtitling industry, but regrettably, this merit turns out to be its Achilles heel. It sacrifices objectivity to some extent and consequently incurs alarming problems; that is, while deploying this model, the evaluators are incapable of avoiding personal preference because, for example, there is no clear delimitation between the severity of errors, and this makes classifying errors becomes a matter of individual choice. Accordingly, it leaves the issue of undue subjectivity of TQA unsolved. Despite its imperfections, the FAR model has been employed to assess the translation quality of culture-bound terms in subtitles [12]. Albeit essentially disconnected from systematic linguistic theories, most empirical models come into use for translator training or examination purposes, such as diagnostic tools, and some have even performed as or have been assimilated into in-house guidelines in the subtitling industry.

By and large, playing an active role at the forefront of the research on the TQA of interlingual subtitling, the empirical models are methodologically homogeneous because most of them can be summarized as introducing 
specific parameters, weight setting, designing procedures, and calculating scores for randomly selected examples. To be clear, the scoring system is not the point of contention, and it is not reasonable to assert the point that error analysis should be rejected in TQA. Rather, it is highlighted that translation quality assessors should shift the locus from simply totaling the number of errors at the word or sentence level and adding penalty points according to some preset standard. To be specific, the crux (and top priority) of a theoretical TQA scheme is to determine whether a translation is problematic neutrally and to curb subjectivity by articulating the reasoning behind the decision, which needs rigorous scientific underpinnings rather than merely pointing out where the faults lie and putting them into several categories.

To bring this overview to a close, inadequacies continue to exist in present-day TQA models for interlingual subtitling and are detrimental to the appraisal of professional subtitlers, and more particularly, to trainee subtitle translators because tolerably objective TQA criteria are indispensable for both rendering feedback and improving their subtitle translation competence. On the one hand, the widely noted divide between academia and industry needs to be bridged. On the other hand, a theoretically robust scheme should be instituted to decide on the fitness of translated subtitles; that is, to construct a systematized TQA model for interlingual subtitling that is grounded on linguistic theories. The current study aims at discussing the possibility of establishing such a theoretical model.

\section{PRELIMINARY CONSIDERATIONS}

When it comes to the discussion of TQA of interlingual subtitling, it is safe to assume that motivation can be drawn from the (linguistically informed) TQA theories on monomodal texts in existence, which can provide the springboard for the development of the TQA model for subtitle translation (since strictly speaking, there is no available theoretical TQA model for subtitling as yet). The current research takes a linguistic approach, which is often adjudged a less valued method in the academic discussion about TQA. Under the global methodological direction of the "cultural turn" in some strands in audiovisual translation studies, such an approach may cause concern among some scholars in this field, who often label it as "partial and inadequate" and renounce any grounding in linguistics. It is argued that what should be rejected is this tendency to dissociate TQA, and broadly speaking, the analysis of translated texts and translation as a whole, from linguistics, because it is also true that "translation is steeped in language and is always - though not exclusively, perhaps not even primarily - a linguistic operation" [13]. This reality provides a rationale for evaluating translation quality from a linguistic perspective and holding an unwavering text-based orientation for the whole enterprise.
To the best of knowledge, erected on Halliday's systemic functional theory (SFT, which informs Systemic Functional Linguistics, SFL), House's systemicfunctional theory of TQA $[4,14,15]$ is one of the most influential and sophisticated TQA framework (for monomodal texts) that maintains a stable linguistic foundation in that it depends largely on the (Hallidayan) contextual analysis and comparison of field, tenor, and mode. Afterwards, further developing House's model, $\mathrm{Si}$ [5] undertakes the construction of the quality assessment model for translation between Chinese and English by incorporating lexicogrammatical analysis, with a focus on the equivalence (delivery) of three metafunctions (i.e., ideational, interpersonal, and textual metafunctions) between source text (ST) and target text (TT). SFT can be applied to translation between English and Chinese in that these two languages have attested to be able to be described theoretically from the systemic functional perspective.

Remarkably, it has been confirmed that SFT, as an applicable theory that remains in constant dialogue with the application, has exhibited its effectiveness for subtitle translation [16] theorizing and modeling, and, theoretically speaking, been able to serve as the foundation of multimodality, which is an academic spinoff of social semiotics [17] and has been on the agenda of SFL for decades. Moreover, it has been argued that Halliday's cardinal insights into the tri-metafunctional configuration of language can be extrapolated to other semiotic resources [18] in multimodal texts, such as audiovisual texts in the discussion. However, three metafunctions are embodied differently in multimodal texts (i.e., representational, compositional, and interactive functions) as distinct to monomodal texts. This signifies that the present TQA schemes are by no means directly applicable to interlingual subtitling, notwithstanding the legitimacy of borrowing from existing TQA theories to afford guidance for the assessment of subtitle translation quality. Nevertheless, SFT is still, in large part, a common theoretical underpinning shared between (the traditional theoretical) TQA and multimodal discourse analysis (MDA), which denotes it is highly likely that they can be integrated. However, it is foregrounded that refinements should be made to certain concepts deriving from TQA and MDA research to optimally adapt to audiovisual texts, which partake of the sui generis multimodal attributes vis-à-vis monomodal texts, as evidenced by the definition of the audiovisual text.

A text is any stretch of contextually embedded language wherein the individual components relate to one another and form a cohesive whole [14, 15]. Likewise, an audiovisual text is a verbal-iconic construct that transmits codified information via acoustic and visual channels, which are constituted by multiple synergizing and interacting semiotic modes referred to as meaningmaking resources (or media), as defined by Chaume [19]. 
Implicit in this definition is that multimodality, which attends to the relationship between the whole gamut of modalities (as traditional forms of communication and representation), features prominently in audiovisual texts. However, at one time, the translation of audiovisual texts was marginalized (or even largely neglected) in diachronic and synchronic translation studies research canon, as those texts cannot be examined based on traditional translation equivalence criteria (i.e., translation proper), which only allowed for the analysis and evaluation of the translation encompassed in multimodal and multimedial texts by excluding nonlinguistic components in the composition of texts and concentrating on language as the central mode (which obscures the fact that those texts are ultimately multimodal). Verbal mono-modality appeared to be the standard form of communication, and it has been pointed out that the dominant research perspective remains largely linguistic [20]. This situation was redressed thanks to the improvement in the theoretical, methodical, and analytical equipment of the discipline of multimodality, which moves beyond the semiotic system of language to include multiple semiotic resources that construe the context of situation and culture, which are themselves multimodal.

Audiovisual texts are multimodal inasmuch as the interaction between subtitles, and other semiotic channels (the articulated and simultaneous deployment of a panoply of semiotic resources or "modes," see [21]) is crucial to the meaning-making of an audiovisual text. Such a semiotic cohesion is defined as a type of relationship "between two or more signs of different or same mode" that operate simultaneously in the production of meaning and enables the viewer to appreciate a coherent multimodal unit. In a given multimodal text, signs are arranged so that the meaning of such a text goes beyond the simple addition of meanings of each element. Considering that the multimodal characteristic arguably intensifies the challenges faced by subtitlers (as well as assessors), it follows that the prerequisite for current exploration is a mode of analysis that paves the way for the functional dissection of semiotic elements in interaction and combination, and the contribution of the visual and verbal modes involved to the meaning of the audiovisual text as an integrated entity in a principled manner.

The translation of an audiovisual text (or unambiguously, its components, subtitles) is a multimodal semiotic act that requires subtitlers to navigate the rich multimodal information in ST - in other words, to weave the new meaning-making resource, i.e., subtitles (as a segment of a dynamic audiovisual text), into the overall semiotic fabric of the film [22]. This multisemiotic (or polysemiotic, see [23]) nature of subtitling $[24,25,26$, to name but a few] sets subtitle translation apart from other translation practices. Subtitle translation is a type of intersemiotic translation, as it involves not only the transfer of linguistically codified messages in the original dialogue (verbal modes) but also information of visual and acoustic information (nonverbal modes) in the (textual and extra-textual) setting of the audiovisual texts. In other words, subtitling goes beyond the translation of the linguistic constituents between two languages and deals with the translation from the text of a film (ST) to the text of a subtitled film (TT) [26]. Although in most cases, subtitlers can manipulate meanings only through the written mode (subtitles, one medial variant of the mode of language), it is possible that meanings can be distributed to all modes involved in the audiovisual text, such as the mise-enscene mode (i.e., the visual mode or image), in the process of subtitle translation [26]. This fact can be supported by the conception of "diagonal translation" [24], based on which subtitling is "diagonal" in that it involves translation from the spoken to the written mode (compared with "horizontal" translation, such as interpretation, which is a transfer within the spoken mode).

There is no gainsaying the fact that all texts (even those that seem to be purely monomodal), as a matter of fact, contain some non-verbal elements (e.g., typology and layout), as the message cannot be rendered without some physical support [25]. Given that "no text is strictly speaking monomodal" [25], all discourses are arguably multimodal: even in the case of a language-based "monomodal" written text, where two semiotic systems, language, and typology are involved in the meaningmaking process. However, in such a text, the system other than language is ancillary to language itself and cannot be independent of it, i.e., typography cannot exist without language [27], and this also holds for audiovisual texts, for instance. In audiovisual translation, the components of audiovisual texts cannot be extracted from one another while the text itself still maintains cohesion [27]. Therefore, the multimodal materiality of audiovisual texts should be brought to the fore in that the verbal modes are not the only element that is at play in the process since the subtitles "are useless just as a text" and "are intended to exist only by being merged with" nonverbal modes [28] that also make a solid contribution to the meaning-making process (via semiotic cohesion or the distribution of meanings between various semiotic resources) and the delivery of communicative intention. In this sense, the audiovisual text is essentially multimodal rather than exclusively verbal [28] and cannot be reduced to the mere sum of its separate modes of meaning-making [21]. This has broader implications to subtitle translation as a form of "multimodal translation." As a matter of fact, [t]ranslating only the linguistic elements without heeding the value of the other semiotic dimensions would certainly be a recipe for disaster [8]. In subtitle translation, what is transferred includes not only the meaning in verbal modes but also information in images. 
From here, it is obvious that what should be forsaken in audiovisual translation studies is the perception that subtitles are only considered as the written counterpart of the verbal (spoken) mode. Therefore, systematic theorization is needed to identify the components in the audiovisual text that influence translation activity and the investigation into the extent of such influence. Accordingly, before assessing the quality of subtitle translation, it is imperative to conduct the multimodal analysis of audiovisual texts as a whole, rather than focusing only on subtitled texts. Moreover, the foregoing TQA models targeted at monomodal texts should be tailored to the need for the analysis of interlingual subtitling in response to the multimodal properties of audiovisual texts.

Admittedly, it is safe to claim that it is impractical to formulate a model that eliminates subjectivity and appeals to everyone's taste since subjectivity is immanently ascribed to TQA, as is a form of value judgment per se. It is even surmised that this problem is to some extent insoluble in a way that is scientifically justifiable [3], but it needs to be reiterated that this does not give rise to the assertion that TQA studies are worthless in terms of translation-critical agnosticism [29], and the quest for a well-engineered model should not be abandoned. TQA cannot and should not be values-free [11]. What is overarching to realize is that it is inadvisable to trade off scientific rigor for feasibility while constructing a TQA model. Hence, this study will mainly focus on the sophistication, not the accessibility of the theoretical model. Moreover, the technical issues (i.e., extralinguistic constraints) of subtitling, such as segmentation and punctuation (but this does not mean that they assume lesser importance in subtitling), will be left undiscussed in this research.

To sum up, it is argued that a theoretical TQA model for interlingual subtitling can be established by integrating MDA and the previous TQA model for monomodal texts $[4,5,14]$. Next, these two theoretical foundations will be introduced in turn.

\section{THEORETICAL FOUNDATION}

\subsection{Assessing Translation Quality from The Perspective of Systemic Functional Linguistics}

Widely acknowledged as one of the most intractable and challenging problems in translation studies, TQA is instrumental in determining the quality of translated texts and has overriding value in the world of professional translation. Thus, in the academic realm, TQA has engaged the attention of theorists who have been motivated to strive for the establishment of a full-blown TQA model from multiple theoretical standpoints, and some of them have invested efforts into assessing translation quality from the perspective of SFL.
As one of the most far-reaching and pioneering theories of evaluating translation quality, House's functional-pragmatic TQA model [2, 4, 14], which is based on contrastive German-English discourse analysis, pragmatic theories of language use, and Halliday's SFT [30], has inaugurated a new era in translation studies. Her TQA model is by its very nature formulated for the analysis of the linguistic-situational features [1] of the source and target (written, not oral) text enveloped in the particular situation. She avers linguistic-textual analysis (translation as linguistic-textual operation, see [4]), and comparison [2] is of primacy in TQA. The touchstone for an adequate translation is the functional-pragmatic equivalence between the original text and translation. This concept of "equivalence," also adopted as the criterion in the current research into the TQA model for interlingual subtitling, is affirmed to be orthodoxy in setting forth the ST-TT relationship and the fundamental criterion of translation quality, which entails the analysis of the function of the text and the ensuing comparison between the function of ST and TT with the deployment of appropriate analytical apparatus.

In view of the fact that a text is intricately interwoven with the context of situation [2], the situationaldimensional features [4], which include the dimension of language user and language use, shape the textual profile of the source and target text and thus should be scrutinized to determine whether the equivalence has been achieved between source and target text in semantic and pragmatic aspects and whether equivalent situational-dimensional means are deployed to achieve this equivalence through linguistic analysis. These aspects dovetail with the Hallidayan ideational and interpersonal metafunction of the language, respectively. Therefore, translation can be construed as the preservation of meaning in the language transfer with the deployment of pragmatic instruments to recontextualize the ST, i.e., reproducing the ST semantically and pragmatically in the target language, or a view of translation as culture-conditioned recontextualization [2]. In keeping with this, the functional match between ST and TT is viewed as the yardstick of TQA.

It is highlighted that in House's TQA scheme, two uppermost parameters, register (which includes various situational dimensions) and genre, have been incorporated to characterize the function of an individual text. The former serves as the linguistic manifestation of the context in which a text lies, while the latter links register to the text's function [14]. It deserves to be noted that the above-mentioned situational dimensions, as manageable components in the analysis, are considered to be the means by which the function of the text is realized and are subsumed under the category of register.

The operation of House's SFL-informed TQA [4, 14] is briefly epitomized as follows. The evaluation begins with the analysis of ST's register components, i.e., field, 
tenor, and mode, which are dissected at the lexical, syntactical, and textual level, and the description of ST's genre, or "deeper textual structures" [2], is provided to expatiate upon ST's function, i.e., to obtain the textual profile that characterizes the function of ST. Next, the textual profile of TT is established with the same parametric system as in the first phase to characterize the TT function. The degree to which the textual profile and function of the translation conform to those of the original is the benchmark for translation quality. Afterwards, on the basis of function-measuring criteria, mismatches between the ST and TT are elaborated, some of which may be warranted to be necessary for translation and thus are conducive to translation quality. As for translation errors, they are subsumed under two categories, dimensional (pragmatic errors) and non-dimensional mismatches [4]. Ultimately the statement of the functional conformity between ST and TT is furnished, which completes the process of a qualitative assessment of translation quality.

Putting the strong accent on the sharp distinction between comparative (linguistic) analysis and (social) judgment, House [2] professes that her TQA model is not inclined to enable evaluators to make a definitive assertion about the TT's translation quality. Instead, the contrastive linguistic analysis only establishes a crucial starting point for the subsequent evaluative judgment. To be exact, the functional-pragmatic approach alone is not sufficient to allow assessors to pass judgments on the quality of translation, as translation decisions never solely rely on the ST and the translator's interpretation and linguistic considerations. As a complement, House [2] proclaimed that other interested parties involved in the translation practice (besides the translator), such as readers, along with a range of non-linguistic social factors (or constraints), figure prominently in translation both as a process and a product.

\subsection{A Multimodal Approach to The Analysis of Subtitle Translation}

The SFL approach is well-established in audiovisual translation studies, especially in subtitling. However, in view of its multimodal nature, the previously mentioned SFL-informed TQA models for monomodal texts are inapplicable to audiovisual products. Further, the tendency to focus exclusively on subtitle texts, which are treated in the same manner as single-modal texts from time to time, should be avoided in subtitle translation quality research. Therefore, a multimodal approach should be adopted to address the TQA of interlingual subtitles as multimodal texts. Several studies have exploited the SFL-driven approach to multimodal textual analysis $[18,30,31]$, and since the aforementioned mainstream theories of TQA are mainly founded on an identical basis, which indicates that SFL is capable of serving as an interface between multimodal analysis and
TQA in the cause of formulating the TQA model for interlingual subtitling.

In one of the most inspiring works in the study of multimodality for translation purposes, Chen [16] approaches English-Chinese subtitle translation from the angle of SFL-based multimodal analysis, which immediately sheds light on the current endeavor to construct a TQA model for interlingual subtitling between English and Chinese. Before the improved framework for MDA (as discussed in the last section) and Chen's approach [16] are pulled together to produce an integrated MDA scheme of the audiovisual text for TQA in the current study.

As it is not viable to attend to all details of her sophisticated model here, only the key points that yield beneficial insights on TQA are addressed. Moreover, it should be emphasized that Chen's [16] analytical scheme is not accepted uncritically and indiscriminately but reshaped to conform to the requirement of the evaluation of subtitle translation quality.

In light of her multimodal approach to subtitle translation [16], meaning-making resources involved in interlingual subtitling to transmit information can be sorted into image (visual or non-verbal message), dialogues (in the verbal source language), and subtitle (in target written language), whose interplay is an essential prerequisite for constituting a multimodal entity and internal coherence of the film narration [16]. It is this characteristic that poses challenges to interlingual subtitling. For example, on some occasions, translation shifts in the verbal content occur in order to remain consistent with images [16]. Undoubtedly, subtitle translation cannot be carried out without considering the coexistence of various modes or scrutinizing their interactions in the construction of meaning. Notwithstanding the intrinsic multimodal nature of subtitling (as a form of multimodal translation or intermodal translation, which exceeds modality limits), it should be highlighted that in subtitle translation, the translation from the verbal source language (ST, or its transcription) into target written subtitles (TT) occupies a pivotal position. In other words, semiotic resources other than words should be seen as complementing verbal messages in the multimodal context, but this does not signify that the relationship between verbal and nonverbal elements is hierarchical, i.e., meaning is orchestrated through the selection and configuration of verbal and visual modes that share equivalent status in this process (see $[16,18]$ ). Furthermore, notwithstanding the multimodal properties of audiovisual texts, it should be highlighted that not all subtitles are strongly related to the modality of image (i.e., heterogeneity of intermodal relations), which denotes the existence of monomodal features in subtitle texts. Thus, despite the fact the multimodal transcription of (the grammar system) of visual images [16] is a well-established tool, when 
assessing translation quality of interlingual subtitling, for the sake of simplicity, it is advisable to carry out the frame-based multimodal transcription of certain scenes only when it is deemed necessary rather than transcribing the entire film, which is both time-consuming and not feasible, especially for long audiovisual texts (as recognized in [32]).

Following the nomenclature initiated by Kress and van Leeuwen's [18] studies on multimodality, Chen [16] categorized three metafunctions as representational (ideational), the compositional (textual), and interactive (interpersonal) functions (metafunctions), which are significant in the analysis of subtitle translation (information flow in audiovisual texts) and are also the terms adopted in this study. It should be mentioned that Chen's [16] model for analyzing subtitle translation based on three multimodal metafunctional meanings will undergo certain modifications to satisfy the analytical requirement in the current endeavor to construct a new TQA model for subtitle translation

The representational metafunction manifests itself in the semiotic interplay between the verbal (subtitle) and the visual (image) modes. It indicates the relations between signs and objects in the world external to the representational system $[16,18]$. The representational meanings of audiovisual texts are examined in stills (or shots), which means that cross-shot interaction is not considered in this facet. In audiovisual texts, the semiotic interplay between visual and verbal modes (dependency between modes) within a single shot can be classified into four types [16]: relay (complement), which indicates that two modes convey complementary (but somewhat different) information; anchorage, which demonstrates that the verbal mode further defines messages contained in the visual mode; moorage, which signifies that the verbal mode is elaborated by information delivered via images; reinforcement (which is preferred to redundancy to avoid the implication of being unnecessary), which betokens the coincidence of messages transferred by the visual and verbal modes. In short, such relations, which signifies that the meaning in a single still is presented simultaneously via visual and verbal channels, can be subsumed into two categories: the verbalized-image denotes that messages contained in the visual mode emerge in subtitles (and thus representational meanings are delivered) if space and time constraints allow for such presentation when certain visual information cannot be traced in source verbal messages; the non-verbalizedimage signifies that visual information possessed by the images does not (but certainly can) surface in the verbal mode when visual and verbal source mode convey more or less the same information [16]. In the final analysis, be it the former or the latter type, the way that the visual mode shapes subtitle translation can be construed by the interactions (i.e., logico-semantic relations) between the visual and the verbal modes [16], which can, in turn, reveal five sets of the relationship between the source and target verbal messages (analyzed in shots or stills): target verbal messages (subtitles) can be the explication (elucidation), extension (information added), contraction (the omission of information), generalization or replacement (by the pronouns, the demonstratives, equivalent expressions, or near-synonyms, which implies the inexistence of exact equivalents, see $[7,16]$ ) of the source ones (which are conveyed through the acoustic channel). The cross-modal interactions are, in essence, visual-verbal cohesion realized by linguistic resources, which includes all expressions that are semantically connected with the visual information. The linguistic processing in subtitling (demonstrated by the relationship between ST and TT) is generally warranted by the (contextual) information derived from the visual mode (i.e., the information flow of film texts), and thus no essential information will be lost during this process.

The compositional metafunction implies that textual meanings are materialized by semiotic cohesion (cohesive relations) between different filmic levels in multimodal texts [18]. Promoting textual integrity by turning individual characterizations of the world into coherent and cohesive unities, the compositional meaning in subtitling is examined in film scenes (or sequences), which implies that the analysis is conducted beyond stills; namely, information both in stills and in preceding or subsequent scenes (i.e., intermodal relations) should be taken into consideration in subtitle translation. Borrowing concepts of anaphora and cataphora in monomodal texts, the semiotic cohesion can be classified into two types of relations respectively: anaphoric (i.e., the information presented in preceding scenes) and cataphoric (i.e., the information that emerges in subsequent scenes) relations [16], which delineates the source verbal information's association with its counterpart (i.e., the above-mentioned explication, contraction, and replacement). As the cohesive relations (or semiotic ties, see [16]) between source verbal language and images should be distinguished from that between the target counterpart (subtitles) and images, the concepts of co-referentiality, co-classification, and coextension are introduced to analyze cross-modal semiotic cohesion to examine anaphora and cataphora in audiovisual texts: co-referentiality denotes the relationship between elements of image and devices of reference (lexis) in a language, i.e., the pronouns and the demonstratives; co-classification is materialized either by ellipsis [33] and in the case of subtitle translation, the elements in images induce a reduction in subtitles; coextension can be constructed via three types of relations, namely synonymy, hyponymy and meronymy [16] between verbal and visual modes. It should be noted that Chen [16] also discusses the dichotomous function of semiotic cohesion, i.e., identity-entitled and logicentitled function, in her analysis of compositional function. Revealing the functions of the aforementioned semiotic cohesive devices, such a division plays a 
significant role in the analysis of compositional meanings, and it is necessary to elucidate those two functions within the framework for the TQA of interlingual subtitling.

The interactive metafunction refers to the relations between the semiotic system (can be a sign or a semiotic mode) and its receiver. In the context of audiovisual texts, this metafunction is built on the semiotic interplay that affects the interactions between viewers and subtitled materials [18]. It is examined with reference to contact, attitude, and distance [16], which are adopted from Kress and van Leeuwen's [18] analytical scheme of interactive meanings in print images. It should be mentioned that Chen's [16] approach to interactive metafunction needs to be adjusted to address the underlying weakness of her framework. The analysis of contact and the so-called information-oriented attitudes (one branch of Chen's [16] analysis of attitude) appears to overlap with those of representational and compositional metafunctions, covering cohesive relations in the visual-verbal system of interlingual subtitled films but in contrasting terms. By contrast, the participant-oriented attitude (the second branch), which originally denotes whether visual participants are directly referred to in subtitles, is considered as simply a matter of personal preference. It arouses suspicion as to whether this concept deserves a special mention in the original framework, and thus it is rearranged to characterize the relationship established between characters (whether they are animate or inanimate) in audiovisual texts as well as its potential effect on subtitle translation (i.e., when translating expressions related to interpersonal meanings in language). In addition, the third aspect, distance, which copes with the tie between images and viewers, merits attention. It is believed that when several types of modes are engaged in transferring approximately equivalent information, i.e., information is conveyed through more than one mode, the distance between viewers and films becomes closer (also known as direct addressing); otherwise, the distance becomes further [16]. However, there are, by far, no apparent reasons to believe that the direct linkage exists between translation quality and variations in distance. Hence, when constructing the TQA model for interlingual subtitling in this study, the interactive metafunction will only be investigated in the sense of participant-oriented attitude.

In sum, this framework, as an integral component of the theoretical TQA model, mainly attends to the investigation of the representational, compositional, and interactive metafunction in audiovisual texts. Serving as the theoretical mainstay of current research, the SFLinformed TQA (for monomodal texts) and the multimodal approach to subtitle translation can be integrated, as shown in the next section.

\section{A TRANSLATION QUALITY ASSESSMENT MODEL FOR INTERLINGUAL SUBTITLING: THEORETICAL DISCUSSION}

It has been highlighted in previous TQA models for monomodal texts that equivalence is strongly significant. In keeping with this, it is essential for an explanatorily adequate TQA theory to specify what type of equivalence is of particular relevance and operationalize this equivalence relation for this study. Following the systemic-functional model developed by House [4, 14], this study attributes the functional theory of translation with functional equivalence as its nucleus to be the mainstay of the new TQA model for interlingual subtitling. It is evident that such a "functional" equivalence requires the TT to be the "functional" equivalent of ST. In keeping with this, the notion "function" is conceptualized to characterize the function of individual texts as the starting point of the evaluation of translation quality. To unveil the quintessence of this "function," how language is modeled in SFT, which is the common theoretical underpinning shared by both TQA and MDA in this research, is in want of further elaboration. While this concept is accorded great importance within the theoretical framework of this research, it goes without saying that it is beyond the scope of a single section to expatiate on such a magnificent architecture of language, but essentials are covered nonetheless.

In SFL, three interdependent levels, or strata, together with the extralinguistic level of context (of situation and culture), figure prominently in the functional approach to language and, accordingly, translation. The three linguistic levels are the stratum of semantics, lexicogrammar, and phonology/graphology [34]. The present discussion concentrates on three strata, context (of situation), semantics, and lexicogrammar, where the features and structures of ST can be preserved in translation. The semantic stratum supports four "functions" or "strands of meaning" [34], experiential, logical, interpersonal, and textual meanings (functions), which are subsumed under three strands of metafunctions [34]: ideational (comprised of experiential and logical meanings) metafunction, which is concerned with the resources for the exchange or human experience [33], interpersonal metafunction, which reflects the interactive function of language and enacts social relations, and textual metafunction, which is oriented to encode the organization and structure of discourse as it unfolds [33, 34], and thus enables the fore two functions. Notwithstanding that ideational meaning is often privileged in translation, there is no hierarchical order between three metafunctions, which are simultaneously manifested in the organization of any (monomodal) text, and broadly speaking, every natural language. Ideational meaning (i.e., meaning as representation) is largely (but 
not exclusively) realized through the grammatical system of transitivity (the process, participants, and the circumstances), and interpersonal meaning (i.e., meaning as exchange) primarily through the mood system, and textual meaning (i.e., meaning as message) mainly through systems of theme (thematic structure) and information as well as through cohesive devices [33]. In other words, the metafunctional meanings of a text can be revealed by analyzing the above systems. These grammatical systems are the main components of the lexicogrammatical stratum, and the level of phonology/graphology represents the stratum of linguistic "expression" [34] as opposed to "content".

As a crucial intermediate level, the semantic stratum is where the lexicogrammatical stratum (or the grammatical system of language) interfaces with the level of context (of situation, or social system), that is to say, the features in the semiotic structure of the context (of situation) "activate" (or create) a regular (but not absolute) semantic configuration (or register, a concept to be clarified below) of the semantic stratum, and correspondingly components of the lexicogrammatical level. It follows that context is in a realizational relation with (or "realized in") language through register conceived as a higher-order configuration at semantic stratum. Such an interstratal relation [30], or referred to as "realization/activation," "activation-construal" relation [35], means that the stratum of context is represented (realized) in different shapes across the other strata [35]. It is noteworthy that this activation-realization dialectic does not extend to the strata below the lexicogrammar. For instance, phonological choices, as an expression form, are not in the realizational relation with lexicogrammatical choices, but a relationship of signaling [35], which means that lexicogrammatical selections specify phonological and graphological elements, not the other way around.

The relationship between language and context can be depicted as the context is realized in language and language construes (a term synonymous with "realize") context [35]. The scale of instantiation indicates that a specific language event constitutes a selection from the language system. A specific context of situation is an instance (the instantiation) of the sociocultural context (i.e., context of culture) [35], and the context of culture is instantiated by a given context of situation, which is in turn realized in an actual text as a semantic unit. Moreover, the context of situation activates, at semantic stratum, a register (as a configuration of linguistic meanings or a continuous process of semantic selection at the lower lexicogrammatical stratum, see [30]), which is instantiated by a text (or a text is an instance of a register). In this way, the relationship between context and text has been theorized to be dialogic [34]: context activates meanings and is itself semiotically construed in language.
Briefly, the kernel of SFT is that the meaning of a text can be interpreted when and only when the context in which it unfolds is involved. In keeping with this, a text, as a linguistic construct of social interaction, is defined as a stretch of contextually embedded language [15]. The function of a text (or the metafunctional meaning of its linguistic components) cannot be identified without appealing to the context of situation in which it operates. Context of situation is often equated with what comes to be known as Register, i.e., the language variety according to use (and by convention developed in previous studies, the "context of situation" and "Register" are used interchangeably in this research, see [15]). In addition, it has been argued that translation is more than the rendering of words by their equivalents in another language, but the placing of linguistic symbols in responsive to the context of situation, with which a text (or an utterance) is interwoven inextricably and from which it can never be detached. Following this argument, replete with contextual knowledge, language (text) becomes meaningless if isolated from perceptible situations, let alone translation as a linguistic operation between two natural languages. Translation is characterized as the recreation of meaning in context through choice in the interpretation of ST and generation of TT. These inferences are principally motivated by the fact that the language people use differs in connection with its condition (situation) of use, i.e., each context of situation commands its own range of linguistic features. It follows that in the TQA model for interlingual subtitling, the function of utterance in source and target language (language in use) must be interpreted and defined by reference to the context of situation before proceeding to the comparative analysis.

Returning to the earlier discussion, the notion of context is related to the functional organization of language-in-text [15], which represents a text's function, and thus occupies a place of prominence in the analysis of translated subtitle texts. To decompose such an abstract concept into manageable components, the description of registers in SFT and the contextual analysis of translated texts $[15,35]$ is introduced and adjusted to construct a scheme for its analysis, which is indispensable in the TQA model for interlingual subtitling. The three contextual constellations, labeled Field, Tenor, and Mode, which compose the register and describe its tripartite sematic configuration, deserve more attention. The three parameters are sketched as follows (adapted from [30]).

The category of Field lends itself to the description of subject matter or "the area of operation" [30] of the social action in which language is an essential component and goal or purpose of this action. Tenor captures the nature of participants, or precisely, the relationship between participants in terms of social status, roles, and distance vis-à-vis one another. Mode refers to the medium of communication (spoken, written, dialogue, monologue mode, or some amalgamation), channel (such as aural or 
visual) through which information can be communicated, and the rhetorical mode, i.e., "the role (function) of language" (in context) to the activity.

As mentioned earlier, Field, Tenor, and Mode refer to the aspects of the situation (i.e., its context of use) in which language-in-use (or text) unfolds and operates and with which linguistic features of a text are correlated. Deployed to capture characteristics of (the context of) situation, these parameters are encapsulated systematically in a text [30]. To further explain this relationship, certain contextual attributes, as suggested by Halliday [30], can be realized systematically in, or "resonate systematically with" [35] language, which is generally known as the "context-metafunction hook-up hypothesis" or the "context-metafunction resonance hypothesis" [35]. Based on this presupposition, Halliday's trinocular semantic systems of metafunctional meanings [33] and correspondingly, three languageinternal metafunctions, which exhibit the organization of the semantic possibilities of language and construe meaning relevant to contextual parameters, will be particularly, although not exclusively, oriented to three categories of contextual configuration respectively: briefly, Field is prone to be construed through ideational meaning, and Tenor through interpersonal meaning, and Mode through textual meaning, or textual and organization resources. In this way, the register is arranged metafunctionally, and it is by virtue of this linkage that translation involves trade-offs in choices across the metafunctional spectrum (and contextual configuration).

It is noted that the context of situation is characterized as a specific instance of a larger system, i.e., context of culture, or Genre, developed by Martin [36] as a higher stratum. Lying behind which every (context of) situation [37], Genre characterizes the meaning potential immanent in a culture [36] and triggers a particular register and the structure of a text as an instance of register. Therefore, in line with register as a higher-order semantic configuration, Genre is in turn characterized as a statement of the potentiality of register choices, or "pattern (configuration) of register patterns" [36], on each stage of a text as it unfolds [38]. It has been highlighted that the analysis of context of situation alone is not sufficient to yield a statement of the function of an individual text [15]. Therefore, both the immediate context of situation and the context of culture in general shall be involved. Following House [15], Genre, as a category above (but of equal importance to) register, is taken into consideration in this study to connect a text to the type of texts with which it shares mutual socially stipulated purposes and similar structures in a specific linguistic and cultural community [15]. In contrast with her breaking down register into manageable components, House does not approach Genre in the same manner. Moreover, she claims that Genre remains to be a socially determined, pre-scientific category and an ambiguous concept "in the sense that its parameters cannot be set by scientific degree" with certain explications (see [4]). Notwithstanding that this assertion is still open to question (since there are some previous studies on Genre in SFL), and the circumvention of in-depth Genre analysis (without even trying) is sure to provoke criticism here, this study determines to acquiesce in House's verdict in consideration of the complexity of dissecting Genre: TT should belong to the same Genre as the ST [4]. This is entirely plausible in the case of interlingual subtitling, through which, by default, the Genre of the audiovisual text cannot be converted into another. In keeping with this, the challenging attempt to deconstruct the category of Genre will not be undertaken in this research

Taken together, context and language constitute a relationship of reciprocal influence, such that language shapes (construes) context as much as context shapes language [15]. More importantly, the stratal attribute of language [34] demonstrates that with context (of situation) as a higher stratum of meaning, the meaning of language (text) resides systematically in all levels and is realized in contrasting forms across strata, in which the meaning potential of semiotic resources are distributed.

Trailing the strand of work that situates translation at the stratum of semantics and context of situation (e.g., House's systemic-functional theory of translation, [4, 15]), this study ascribes particular significance to three aspects of meaning in translation: semantic, pragmatic, and textual meanings, which dovetail with three metafunctions developed in SFT. The linguistic features, actualized in the lexicogrammatical stratum, simultaneously resonate with the tripartite dimensions of register variation, i.e., Field, Tenor, and Mode. Ergo, this register-metafunction solidarity reveals the essence of the aforementioned "functional" equivalence: an adequate translation can be conceptualized as the replacement of a text in a source language by a semantically and pragmatically (and textually, without a doubt) equivalent text in a target language $[4,15]$. This denotes the maximal preservation of the triumvirate of metafunctional meanings in translation [15], i.e., the ideational meanings (of ST) are reconstrued, interpersonal meanings (of ST) are reenacted, and textual meaning (of ST) is represented in TT. It follows that the "equivalence," or optimum approximation, between the contextual configurations of ST and TT, can be observed in the comparative analysis of register, i.e., the relative stability of the register in the process of translation can be defined as a default criterion, which means that has been argued that more often than not, the register of the ST will not undergo drastic modification in translation proper. Such a transformation, if occurs, actually represents the degradation of ST constraints; that is, the more register variables alter, the more likely that the resultant (target) text will not be a translation in the narrow sense. It is on this account that 
translation errors can be detected through register analysis.

It is tried and true that though largely developed for the analysis of discourse systems in language, SFT (without any major adjustments, see [16]) can also serve as the central theoretical framework for the analysis of multimodal texts (also as constructs of meaning), or specifically speaking, systemic functional multimodal discourse analysis (SF-MDA) [18], which is grounded on Halliday's premise for SFT that the orchestration of semiotic resources (including language) in a text demonstrates the social function of both itself and its components. Therefore, the principle that "[c]ontext determines systems in language, but it is also construed by them" [34] can be extended to encompass the multimodal texts, which are also in a realizational relation with their multimodal context of situation. Stretching beyond the established systemic-functional analysis of linguistic resources, the SF-MDA approach generally adheres to the fundamental conception and analytical tools (stratification, notably) fostered in SFT. Of crucial importance here is that the functionalities and underlying organization of various semiotic resources in multimodal texts are also theorized metafunctionally (as in their monomodal counterparts, i.e., linguistic texts) within the framework of SF-MDA. This convergence of metafunctional diversity allows semiotic modes in multimodal texts to fulfill, though differentially, three metafunctional meanings as well. It is around these three semiotic metafunctions that multimodal texts are organized.

SF-MDA provides a potent apparatus to enact the extension of the foregoing discussion on the TQA of monomodal texts, mutatis mutandis, to the quality assessment of translation involved in a multimodal context. The semantic interdependency between metafunctional meanings in SFT can be transferred securely to their counterparts, which are simultaneously constructed in multimodal texts (within the framework of SF-MDA), viz. representational meanings (corresponding to ideational meanings) that present informational contents, interactive meanings (converted from interpersonal relations) that reflects interpersonal relations, and compositional meanings that reveal intermodal connections transferred from cohesion and coherence in verbal texts. It is evident that the conspicuous distinction between language and other semiotic resources necessitates this conversion.

To sum up, to elucidate and compare the register profile of ST and TT to be evaluated is of uppermost priority in TQA of interlingual subtitling, on account of the fact that such an operation, as a top-down approach, concentrates on the functional correlation between the configuration of the (context of) situation and the linguistic means deployed in the text. This register analysis provides evidence for the subsequent lexicogrammatical (metafunctional) analysis, which is involved as a bottom-up approach to investigate metafunctional strands of meaning and their contribution to the function of an individual text as a whole. Considering that in the light of SFT, translation (of monomodal texts) is perceived as a process of simultaneous choices in ideational, interpersonal, and textual meanings, metafunctional analysis (of ST and TT) can reveal shifts (in terms of metafunctional meanings) and underlying errors that occur in translation, i.e., whether functional equivalence has been achieved. Against the backdrop of (translated) subtitles as a component of an audiovisual text, the TQA of interlingual subtitling requires considerations beyond the scope of metafunctional analysis in previous TQA models targeted at monomodal texts (which does not necessarily mean that the traditional metafunctional analysis is completely abandoned in the new model, more detail in the next section). Briefly, the metafunctional analysis (in the context of intersemiotic analysis) of the audiovisual text shall probe into three semiotic metafunctions that characterize the meaning-making of semiotic resources that are involved in such a multimodal ensemble. In the last analysis, the theoretical model for interlingual subtitling should embrace both register and (multimodal) metafunctional analysis, which are both indispensable constituents of the discourse analysis of audiovisual texts. In this way, it is confirmed that the theoretical TQA model for interlingual subtitling can be established through the integration of SFL-based TQA (of monomodal texts) and the SFL-informed scheme for analyzing subtitle translation.

\section{CONCLUSION}

Over the past few decades, the persisting demand for a practical and relatively objective TQA model for interlingual subtitling has evoked a wide range of scholarly discussions. To the best of our knowledge, this study is among the first few to discuss the possibility of constructing a theoretical TQA model for pre-recorded interlingual subtitling and makes a preliminary academic contribution to some sporadic research in this direction, and broadly speaking, to audiovisual translation studies. The establishment of a viable conceptual model for evaluating the translation quality of interlingual subtitling not only requires the examination of the subtitle texts exclusively but also entails an inclusive analysis of the entire audiovisual text owing to its multimodal characteristics. Theoretical speaking, it is possible to integrate the existing TQA models for monomodal texts and the analytical scheme of multimodal translation in an attempt to construct a theoretical TQA model for interlingual subtitling.

It should be aware that the theoretical TQA model for interlingual subtitling also has a few shortcomings, but they are not beyond remedy. First and foremost, 
consistent with the earlier mentioned FAR model [10], the detection and analysis of errors in TT lie at the heart of the theoretical model. Therefore, the strong points in a translation are absent from the evaluators' attention. However, it ought to be highlighted that the accurate delivery of ST's information is the basic requirement for a translation task. The merits of a translation can be encompassed in translation criticism, but they are dispensable for TQA. Whether the virtue of a translation needs to be involved in TQA is contingent upon the translation brief, and thus, the present scheme is subject to adjustment in this sense. Next, this model is at the early stage of theoretical conception and has yet to take shape. When it is established in the future, empirical confirmation that involves a higher number of samples should be conducted to validate this model under varying circumstances. What is more, the evaluation of the translation of lyrics, which is frequently encountered in subtitle translation, is not dealt with in the model and needs further probing. Finally, and perhaps most saliently, the weighting of each category of errors that reflects their differential influence on viewers is left undiscussed. To address this issue, eye-tracking and comprehension experiments can be administered in future projects.

Regardless of the limitations, the findings of this study offer much food for thought. Research on TQA is still in its infancy in audiovisual translation studies. This study only presents theoretical reflections to construct a TQA model for interlingual subtitling, and the discovery heralds the necessity for future investigation.

\section{AUTHORS' CONTRIBUTIONS}

Zhen $\mathrm{Hu}$ contributed to the conception of the study and wrote the manuscript.

\section{ACKNOWLEDGMENTS}

The author wishes to thank his parents for their emotional support in the process of writing this article.

\section{REFERENCES}

[1] S. Colina, Translation quality evaluation: Empirical evidence for a functionalist approach, The translator 14 (1) (2008) 97-134.

[2] J. House, Translation quality assessment: Linguistic description versus social evaluation, Meta 28 (2) (2001) 227-257.

[3] G. McAlester, The Evaluation of Translation into a Foreign Language, in: C. Schäffner, B. Adab (Eds.), Developing Translation Competence, John Benjamins Publishing, Amsterdam, 2000, pp. 229241.

[4] J. House, Translation Quality Assessment: Past and Present, Routledge, New York, 2015.
[5] X. Z. Si, Translation Studies from the Perspective of Systemic-functional Linguistics-Constructing a Translation Quality Assessment Model, Foreign Language Teaching and Research Press, Beijing, 2016.

[6] M. Madkour, The application of functional linguistic models for assessing quality of translation: a descriptive analytical study, International Journal of English Linguistics 6 (6) (2016) 87-117.

[7] J. Díaz-Cintas, A. Remael, Audiovisual translation: subtitling, Routledge, London, 2014.

[8] J. Díaz-Cintas, Introduction: audiovisual translation: an overview of its potential, in: J. Díaz-Cintas (Ed.), New Trends in Audiovisual Translation, Bristol: Multilingual Matters, Bristol, 2009, pp. 1-20.

[9] L. Bywood, P. Georgakopoulou, T. Etchegoyhen, Embracing the threat: machine translation as a solution for subtitling, Perspectives 25 (3) (2017) 492-308

[10] J. Pedersen, The FAR model: assessing quality in interlingual subtitling, Journal of Specialised Translation (28) (2017) 210-229.

[11] M. Williams, The assessment of professional translation quality: Creating credibility out of chaos, TTR: traduction, terminologie, rédaction 2 (2) (1989) 13-33.

[12] N. M. Abdelaal, Subtitling of culture-bound terms: strategies and quality assessment, Heliyon 5 (4) (2019) e01411.

[13] E. Di Giovanni, Shifts in audiovisual translation for children: reviving linguistic-driven analyses, in: E. Di Giovanni, E. Chiara, P. Roberta (Eds.). Écrire et traduire pour les enfants: Voix, images et mots, Peter Lang, Bruselas, 2010, pp. 303-320.

[14] J. House, Translation Quality Assessment. A Model Revisited, Gunter Narr, Tübingen, 1997.

[15] J. House, Text and context in translation, Journal of pragmatics 38 (3) (2006) 338-358.

[16] Y. Chen, Translating Film Subtitles into Chinese: A Multimodal Study, Springer, Singapore, 2019.

[17] L. Pérez-González, Multimodality in translation and interpreting studies, in: S. Bermann, C. Porter (Eds.), A companion to Translation Studies, John Wiley \& Sons, Chichester, 2014, pp. 119-131.

[18] G. Kress, T. van Leeuwen, Reading Images: The Grammar of Visual Design, Routledge, London, 2006.

[19] F. Chaume, Synchronization in dubbing: a translational approach, in: P. Orero (Ed.), Topics in 
Audiovisual Translation, John Benjamins Publishing, Amsterdam, 2004, pp. 35-31.

[20] H. Stöckl, In between modes: Language and image in printed media, in: E. Ventola, C. Charles, M. Kaltenbacher (Eds.), Perspectives on multimodality, John Benjamins Publishing, Amsterdam, 2004, pp. 9-30.

[21] A. Baldry, P. J. Thibault, Multimodal Transcription and Text Analysis, Equinox, London, 2006.

[22] L. Pérez-González, Appraising dubbed conversation: Systemic functional insights into the construal of naturalness in translated film dialogue, The Translator 13 (1) (2007) 1-38.

[23] H. Gottlieb, Language-political implications of subtitling, in: P. Orero (Ed.), Topics in audiovisual translation, John Benjamins, Amsterdam, 2004, pp. 83-100.

[24] H. Gottlieb, Subtitling: diagonal translation, Perspectives 2 (1) (1994) 101-121.

[25] P. Zabalbeascoa, Dubbing and the nonverbal dimension of translation, in: F. Poyatos (Ed.), Nonverbal Communication and translation, John Benjamins Publishing, Amsterdam, 1997, pp. 327326.

[26] Y.T. Chuang, Subtitling as a Multi-modal Translation, in: G. C. F. Fong, K. K. Au (Eds.), Dubbing and Subtitling in a World Context, Hong Kong: Chinese University Press, Hong Kong, 2009, pp. 79-90.

[27] S. Dicerto, Multimodal Pragmatics and Translation, Springer International Publishing AG, Zurich, 2018.

[28] H. Stöckl, The language-image-text-Theoretical and analytical inroads into semiotic complexity, AAA: Arbeiten aus Anglistik und Amerikanistik 34 (2) (2009) 203-226.

[29] W. Wilss, The Science of Translation: Problems and Methods, John Benjamins Publishing, Amsterdam, 1982.

[30] M.A.K. Halliday, Language as Social Semiotic: The Social Interpretation of Language and Meaning, Edward Arnold, London, 1978.

[31] C. Jewitt, J. Bezemer, K. O’Halloran, Introducing Multimodality, Routledge, New York, 2016.

[32] C. Taylor, The multimodal approach in audiovisual translation, Target: International Journal of Translation Studies 28 (2) (2016) 222-223.

[33] M. A. K. Halliday, R. Hasan, Language, Context and Text: Aspects of Language in a Social-Semiotic Perspective, Deakin University Press, Victoria, 1985.
[34] W. L. Bowcher, Context and Register, in: G. Thompson, W. L. Bowcher, L. Fontaine, D. Schönthal (Eds.), The Cambridge handbook of systemic functional linguistics, Cambridge University Press, Cambridge, 2019, pp. 126-137.

[35] R. Hasan, Towards a paradigmatic description of context: systems, metafunctions, and semantics, Functional Linguistics 1 (1) (2014) 1-32.

[36] J. R. Martin, English Text: System and Structure, John Benjamins Publishing, Amsterdam, 1992.

[37] B. Malinowski, Coral Gardens and Their Magic, Allen and Unwin, London, 1935.

[38] J. R. Martin, Modelling Context: A Crooked Path of Progress in Contextual Linguistics, in: M. Ghadessy (Ed.), Text and Context in Functional Linguistics, John Benjamins Publishing, Amsterdam, 1999, pp. 25-34. 